\title{
Multivariable Control of a Lighter than Air System
}

\author{
Jonathan Samson \\ University of Strathclyde, Royal College Building \\ 204 George Street, Glasgow, G1 1XW \\ jonathan.samson@strath.ac.uk
}

\author{
Reza Katebi \\ University of Strathclyde, Royal College Building \\ 204 George Street, Glasgow, G1 1XW \\ r.katebi@strath.ac.uk
}

\begin{abstract}
This paper describes the control theory behind a new group of wind energy systems known as Airborne Wind Energy Systems (AWES). The control architecture of a kite system and rigid body system is explored and this is then compared to a lighter than air system developed by Altaeros Energies. The plant model of this system is described in detail and the main dynamics of the system are outlined. This paper concludes with a simulation study looking at pitch and altitude control using a multivariable PID control strategy. This was developed using the Pettinen-Koivo method and tuned on a linearized model of the Altaeros System. Results show good tracking in both altitude and pitch set-points.
\end{abstract}

Keywords - AWES, wind energy, lighter than air, control, aerodynamics, PID tuning

\section{INTRODUCTION}

Wind Energy is currently the most mature renewable energy technology available today. As conventional horizontal axis turbines mature, a greater understanding is developed of key components and operational strategies of the system. This has helped to realize the designs of $7 \mathrm{MW}$ machines and beyond. However, on a smaller scale $(2 \mathrm{~kW}-200 \mathrm{~kW})$ there exists an opportunity to develop a novel wind energy system that will alleviate the dependence on both the tower and foundations, as well as offering a potential increase in capacity factor compared to a similarly rated horizontal axis turbine.

The novel wind energy system in question is known as an Airborne Wind Energy System (AWES). These systems use tethers to attach the system to the ground and are designed to attain heights varying between $100-600 \mathrm{~m}$ depending on tether length. There are valid reasons for designing systems to reach these heights as it is known that at higher altitudes the wind profile is more consistent and less turbulent than at lower altitudes due to the fact that there is less interaction with surface ground effects. As proof a study was done which demonstrates that the wind power density was 5 times at altitudes greater than $600 \mathrm{~m}$ relative to ground level [1].

This paper will evaluate each type of AWES and then develop a multi-variable PID linear controller derived from a nonlinear model of a lighter than air system developed by Altaeros Energies. The paper is arranged as follows. Section II will provide an overview of the control strategies implemented thus far in high altitude systems. Section III and IV will focus on the plant model of the Altaeros Energies system and system nonlinearities. Section $\mathrm{V}$ will then discuss how the linear model is derived and how a simple low-level PID optimization is developed to control both the altitude and pitch simultaneously. Results are then presented in Section VI and concluding remarks are given in Section VII.

\section{AwE CONTROL ARCHITECTURES}

There are three types of AWE system namely; a kite system, a rigid body system and a lighter than air system. Each type of system has tethers that tie the system to ground. However, a difference exists in the way that they generate electricity. The kite has tethers that are wound round a mechanical drum that is connected to an electrical generator. As the lift on the kite increases, this translates into high traction forces. The tether, as a by-product of this traction force, is reeled out, rotating the mechanical drum and subsequently generates electricity [2]. Conversely, the other two types of system, the rigid body and the lighter than air system have rotors fixed onto the airborne structure. This may pose serious safety concerns when compared with the kite system.

Both the kite and the rigid body system rely on dynamic control to generate electricity. The kite system is flown in a figure of eight whilst the rigid body flies in a circular pattern. The lift of the kite is regulated through changes of roll angle controlled by the difference in length of the two tethers. The kite is continuously moving throughout the operational cycle and thus the control algorithms employed must be sufficiently fast to account for any change in dynamics that may occur. The rigid body system differs slightly as it has rotors fixed onto the structure [3]. This offers a higher degree of stability as power can be delivered to the rotors changing its operation to that of a propeller. This allows the system to 'hover' in order to gain stability before it enters flight mode. In both cases, the system is controlled to fly crosswind in order to maximize the potential energy capture. This is a well-known result as crosswind flight will increase the apparent wind speed over the aerofoil thus increasing the aerodynamic force and therefore power [4]. The kite and rigid body system along with their typical flight paths are illustrated in Figure 1.
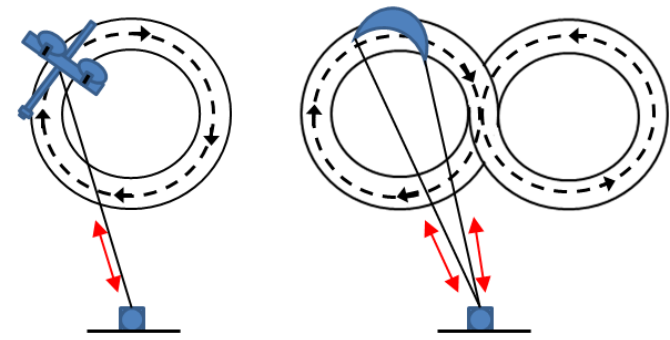

Figure 1 - Flight path of Rigid Body (Left) and Kite (Right) [2] 
The control of these types of system has been well documented in [5-8]. However, the main control challenges associated with these types of system will only be briefly discussed here in order to place the challenges in an overall context with a lighter than air system. The dynamics of the kite and rigid body system are formed through standard Euler equations of motion [9]. This takes into account the lift and drag over both the aerofoil and the tether, as well as determining the mechanical power generated from the unwinding of the tether. The roll angle acts as the control input for the standard optimization algorithm and is controlled by varying the difference in tether lengths. The model for the kite and rigid body system is of a standard form where $x(t)$ defines the state vector of the system, $u(t)$ is the control input and $w(t)$ is the disturbance profile.

$$
\dot{x}=f(x(t), u(t), w(t))
$$

Non-linear model predictive control methods have been employed using (1) in order to optimize both the flight path and power output for the kite or rigid body application [10]. However, a potential difficulty arises when the optimization results in the kite operating at a maximum lift/drag ratio during periods of generation. The danger of this is that the kite could become unstable thus impacting the robustness and overall performance of the system.

In contrast, the third type of high altitude system, a lighter than air system does not need to be dynamically controlled to follow a particular flight path in order to efficiently generate electricity. The shroud filled with helium provides a significant buoyancy force that exceeds the overall weight of the system. This means that under periods of little or no wind the shroud should remain stable in the air. This is compared to the kite/glider system which relies on the lift generated by the aerofoil. The overall controller for the lighter than air system still shares common goals with the other systems but will not rely on a particular flight path for optimality. This is important as it won't be burdened by operating at extreme bounds of stability and will therefore result in a more sustained operation over a longer period of time. Table 1 summarizes the advantages and disadvantages of each system.

Table 1 - Comparison of High Altitude Systems [11]

\begin{tabular}{|c|c|c|}
\hline $\begin{array}{c}\text { Type } \\
\text { of } \\
\text { System }\end{array}$ & Advantages & Disadvantages \\
\hline Kite & $\begin{array}{l}\text { Light-weight } \\
\text { Flexible } \\
\text { Low airborne costs }\end{array}$ & $\begin{array}{l}\text { Complex trajectory } \\
\text { High lift to drag ratios } \\
\text { Cyclic power } \\
\text { generation }\end{array}$ \\
\hline $\begin{array}{l}\text { Rigid } \\
\text { Body }\end{array}$ & $\begin{array}{c}\text { Light-weight } \\
\text { More actuators } \\
\text { available to provide } \\
\text { aerodynamic control }\end{array}$ & $\begin{array}{l}\text { Complex trajectory } \\
\text { Difficult to model } \\
\text { High airborne costs }\end{array}$ \\
\hline $\begin{array}{c}\text { Lighter } \\
\text { than } \\
\text { Air }\end{array}$ & $\begin{array}{c}\text { Stationary } \\
\text { Excellent scalability } \\
\text { Augmented Flow }\end{array}$ & $\begin{array}{c}\text { Increased weight/safety } \\
\text { risk } \\
\text { High airborne costs } \\
\text { Helium requirement }\end{array}$ \\
\hline
\end{tabular}

\section{A LIGHTER THAN AIR SYSTEM}

The concept of using a balloon or a lighter than air system for sensing and data collection is well known [12]. However, as the emerging market for new innovative wind energy concepts took hold it became clear that a buoyant, inflated structure could facilitate electricity generation if a rotor was appropriately attached. This inflated shroud was developed by Altaeros Energies and provides the focus for this paper. The system is comprised of three main components. A shroud with a standard turbine rotor fixed within its shell, 3 tethers connecting the system to ground and a rotating base station that has 3 motor winches that can regulate tether length [13]. An illustration of the system is shown in Figure 2. The left hand picture introduces the Cartesian frame of reference used to relate the shrouds position to the base stations. Note that on the body fixed axis on the shroud three Euler Angles help describe the shrouds position in roll, pitch and yaw. The right hand picture is a real life illustration of the system during testing:
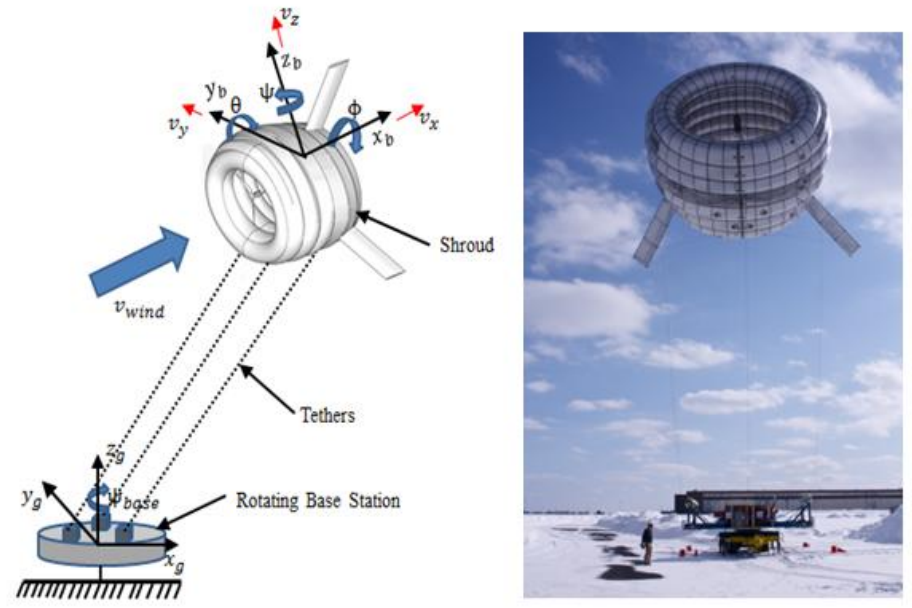

Figure 2 - Altaeros Energies System [16]

Figure 2 shows the Altaeros Energies System during testing (RIGHT) and the relationship between the systems body fixed axis on the shroud relative to the ground (LEFT). The shroud will move in 6 degrees of freedom. There are three translational velocities $u, v$ and $w$ and three rotational velocities $p, q$ and $r$ defined with respect to the body fixed axis $\left(x_{b}, y_{b}, z_{b}\right)$ of the shroud. The angular rates can then be related to the attitude rates in roll $(\varphi)$, pitch $(\theta)$ and yaw $(\psi)$ of the shroud according to the following relationships.

$$
\begin{gathered}
\dot{\phi}=p+q \sin \phi \tan \theta+r \cos \phi \tan \theta \\
\dot{\theta}=q \cos \phi-r \sin \phi \\
\dot{\psi}=(q \sin \phi+r \cos \theta) \sec \theta
\end{gathered}
$$

This result relates a body fixed axis to an inertial reference frame in terms of the three Euler angles. This is well known and follows standard aircraft theory. The translational and rotational rates are combined with Euler's equations of motion to compute the accelerations in 6 degrees of freedom. These 
rates can then be related to the ground fixed axis $\left(x_{g}, y_{g}, z_{g}\right)$ through standard Euler rotation matrices. The equations of motion in both the translational and rotational directions are shown in equations (4) - (9).

$$
\begin{gathered}
\dot{u}=\frac{1}{m}\left(X_{a}+X_{b}+X_{g}+X_{T}\right)+(v r-w q) \\
\dot{v}=\frac{1}{m}\left(Y_{a}+Y_{b}+Y_{g}+Y_{T}\right)+(w p-u r) \\
\dot{w}=\frac{1}{m}\left(Z_{a}+Z_{b}+Z_{g}+Z_{T}\right)+(u q-v p) \\
\dot{p}=\frac{1}{\overline{I_{x}}}\left(L_{a}+L_{b}+L_{g}+L_{T}-q r\left(I_{z}-I_{y}\right)\right) \\
\dot{q}=\frac{1}{\overline{I_{y}}}\left(M_{a}+M_{b}+M_{g}+M_{T}-p r\left(I_{x}-I_{z}\right)\right) \\
\dot{r}=\frac{1}{\overline{I_{z}}}\left(N_{a}+N_{b}+N_{g}+N_{T}-p q\left(I_{y}-I_{x}\right)\right)
\end{gathered}
$$

Together, equations (4) - (9) determine the motion in six degrees of freedom of the lighter than air system. The sum of axial forces in the $x, y$ and $z$ direction are denoted as $X, Y$ and $Z$ with contributions from aerodynamics, buoyancy, gravity and tethers being denoted with subscripts $a, b, g$ and $T$ respectively. Similarly, the moments are given by $L, M$ and $N$. The control inputs are given by $X_{T}, Y_{T}, Z_{T}, L_{T}, M_{T}$ and $N_{T}$.

The force equations corresponding with the equations of motion developed in (4) to (9) are now given below

\section{Aerodynamic Forces}

$$
\begin{gathered}
X, Y, Z=\frac{1}{2} \rho v_{\text {app }}{ }^{2} A_{\text {throat }} C_{D, S, L} \\
L, M, N=\frac{1}{2} \rho v_{\text {app }}{ }^{2} A_{\text {throat }} d_{\text {ref }} C_{M x, M y, M Z}
\end{gathered}
$$

Using equations (10) and (11) the axial forces and moments due to drag, sideslip and lift are computed in the $x, y$ and $z$ direction respectively. $A_{\text {throat }}$ denotes a characteristic reference area of the shroud used for aerodynamic calculations, $d_{r e f}$ is a reference distance, $\rho$ the density of air whilst $v_{a p p}$ is the apparent wind speed incident on the shroud as a function of the wind and shrouds translational velocity.

\section{Buoyancy and Gravitational Forces}

$$
\begin{gathered}
F_{B}=V\left(\rho_{\text {air }}-\rho_{g}\right) \\
F_{g}=-m g
\end{gathered}
$$

Equations (12) and (13) determine the buoyancy and gravity force on the shroud in the body fixed axis. This is related to the fixed inertial reference frame through appropriate Euler rotations to provide buoyancy and gravity contributions in the $x, y$ and $z$ directions. This is denoted as $X_{g}, Y_{g}$ and $Z_{g}$ for gravity and $X_{b}, Y_{b}$ and $Z_{b}$ for buoyancy. The buoyancy force is calculated with the product of the volume of the shroud $\mathrm{V}$ in conjunction with the relative difference between air density $\rho_{\text {air }}$ and the gas density $\rho_{g}$. The gas used in this case was helium.

\section{Tether Tension}

$$
T_{i}=A E \varepsilon \quad \varepsilon=\frac{\left(l^{i}-l_{u}^{i}\right)}{l_{u}^{i}}
$$

Equation (14) determines the tension in node $\mathrm{i}$ of the system. $A$ is the cross-sectional area of the tether and $E$ is young's modulus of the material. $\varepsilon$ denotes the difference in length between the stretched length of the tether $l^{i}$ i.e. the absolute length that the tether currently sits at and the unstretched length $l_{u}^{i}$ which is regulated by the winch speed of independent motor $i$ located on the base station [12]. The tether tension is constrained to being positive at all times. Equation (15) combines the contribution from each tether. Applying appropriate Euler rotation matrices the control inputs $X_{T}, Y_{T}, Z_{T}, L_{T}, M_{T}$ and $N_{T}$, in an inertial frame of reference are determined.

$$
T=\sum_{i=1}^{3} T_{i}
$$

\section{SYSTEM DYNAMICS}

The speed of the three motor winches located on the base station form the control inputs to the system. Depending on the difference in reel in/out speed of each tether the shroud will be controllable in 6 degrees of freedom. The shroud itself is aerodynamically designed to compensate for the high drag that will be present on the shroud at wind speeds typically associated with maximal power generation $(10 \mathrm{~m} / \mathrm{s}-15 \mathrm{~m} / \mathrm{s})$. The system will also be adversely affected by the drag on the tethers and therefore must be designed to have as small an impact as possible. This means it requires a low diameter but with a suitably high strength to weight ratio. The block diagram of the plant model is shown in Figure 3: 


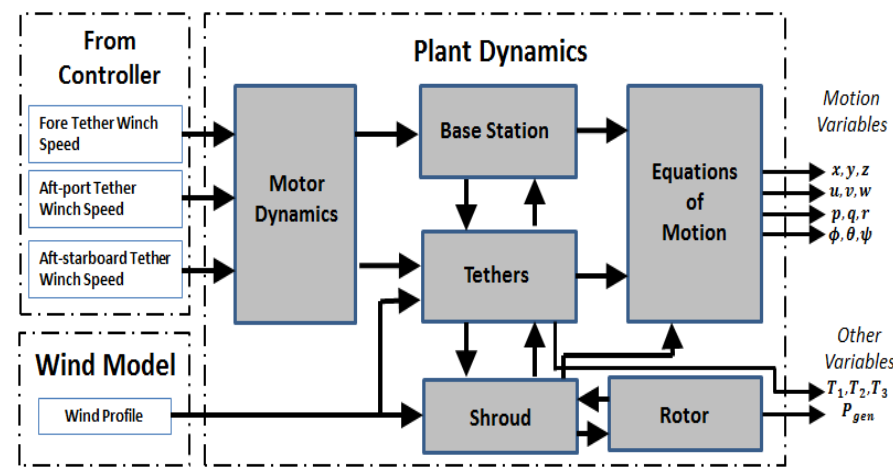

Figure 4 - Plant Dynamics Altaeros Energies System

Figure 3 introduces the plant dynamics of the system with each block corresponding to individual sub-systems created within a model in MATLAB/SIMULINK. The main inputs to the model include the three motor winch speeds to the three tethers and a wind model. For a description of the wind model employed see [13] The three tethers are attached at different points to the shroud to facilitate $6 \mathrm{DOF}$ control. One tether is located at the fore of the shroud and the other two tethers are located at the aft, one on the port side and the other on the starboard. The arrows indicate interactions between each of the subsystems.

The key dynamics of the system reside at shroud level. The position and the stability of the shroud will be important for the successful operation of the system. Figure 4 shows a free body diagram of the shroud with distances $a, b$ and $c$ marked from the center of gravity to the tether attachment points. In turn, the offsets $\Delta x$ and $\Delta z$ describe the position of the center of buoyancy in relation to the center of gravity.

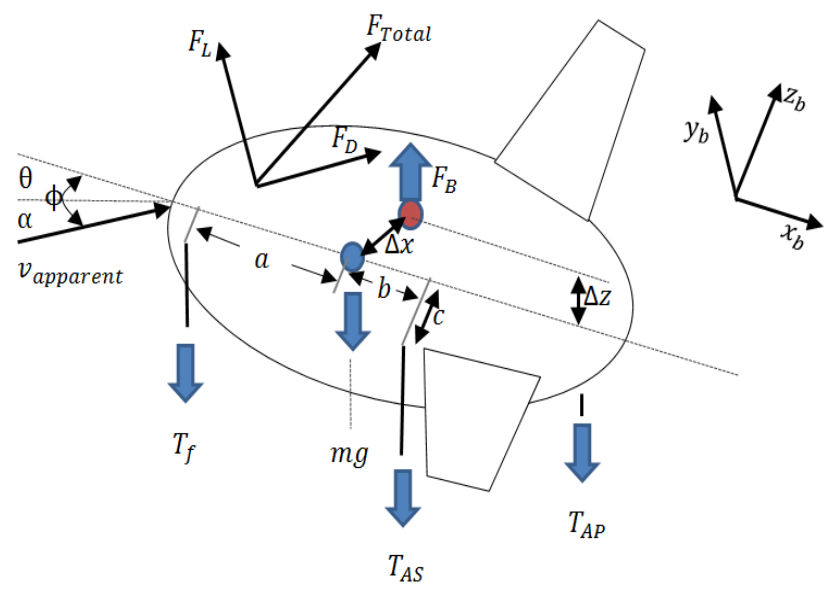

Figure 3 - Shroud Dynamics

There will be mutual interaction between the aerodynamics on the rotor, fixed within the shrouded shell, and the shroud itself. This will affect performance in a positive or negative way. The shroud itself is has to produce lift in order to prevent the system being subject to blowdown induced by aerodynamic drag at higher wind speeds. The next section discusses the relationship between the aerodynamics on the shroud relative to the buoyancy force produced from the employed gas.

\section{A. Aerodynamic Nonlinearities}

The aerodynamics are a function of the angle of attack which is determined by the pitch angle of the shroud relative to the oncoming wind flow. There will be a natural drag on the shroud during normal operation which will lead to instability if not addressed. To address this problem the shroud is designed to produce a significant amount of aerodynamic lift to add to the existing buoyancy force. This has been analyzed in [14], where it was shown that a force ratio relating both the buoyancy and aerodynamic force can be related to the physical parameters of the shroud. The consequence of this from a control perspective is that specific buoyancy to aerodynamic transitions can be characterized as a function of the shrouds geometry. This means a controller could be developed to help smooth the transition between buoyant driven behavior and aerodynamic driven behavior. Two force ratios can be described to illustrated this point, these are shown in equations (16) and (17).

$$
\begin{aligned}
& \frac{F_{B}}{F_{L}}=\frac{2 l g}{v_{\text {app }}{ }^{2} C_{L}}\left(1-\frac{\rho_{\text {gas }}}{\rho_{\text {air }}}\right)\left(\frac{A_{\text {shroud }}}{A_{\text {throat }}}\right) \\
& \frac{F_{B}}{F_{D}}=\frac{2 l g}{v_{\text {app }}{ }^{2} C_{D}}\left(1-\frac{\rho_{\text {gas }}}{\rho_{\text {air }}}\right)\left(\frac{A_{\text {shroud }}}{A_{\text {throat }}}\right)
\end{aligned}
$$

The pitch angle is inherently linked to the lift and drag coefficients computed as a function of the reference area used for aerodynamic calculations $A_{\text {throat }} \cdot \rho_{\text {gas }}$ is the density of buoyant gas used, in this case helium was used but hydrogen was also included in the study for comparison. $A_{\text {shroud }}$ is the area of the shroud filled with helium and $l$ is the length of the shroud. At a particular area ratio $\frac{A_{\text {shroud }}}{A_{\text {throat }}}$ there will be a transition from buoyant to aerodynamic behaviour. Figure (5) shows a force ratio plot as a function of wind speed. Aerodynamic transition points are defined at $4 \mathrm{~m} / \mathrm{s}$ and $6.6 \mathrm{~m} / \mathrm{s}$ for a system filled with helium and $4.23 \mathrm{~m} / \mathrm{s}$ and $6.81 \mathrm{~m} / \mathrm{s}$ for a system filled with hydrogen as a function of lift and drag respectively. These results were taken at an area ratio of 0.2 .

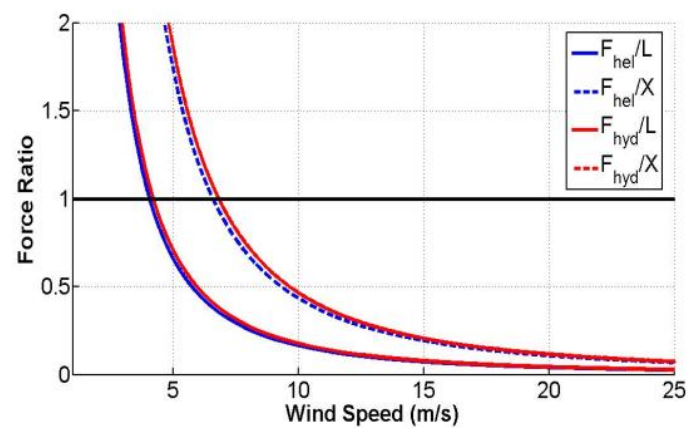

Figure 5 - Force Ratio v Wind Speed

From Figure 5 it is seen that at a force ratio of less than 1 aerodynamic behavior dominates. The system will therefore only be driven by buoyancy at low wind speeds so aerodynamic nonlinearities must be accounted for over the full operating wind envelope, typically $4 \mathrm{~m} / \mathrm{s}-25 \mathrm{~m} / \mathrm{s}$. 


\section{CONTROl OF A Lighter Than Air System}

Autonomous control of this system is imperative if it is to be cost competitive against conventional wind generation. The system must be robust against all aerodynamic nonlinearities which come from the stochastic nature of the wind i.e. wind gusts. The nonlinear equations of motion for this system were set out in Section III, shown in equations (4) - (9), with the control inputs indicated as the combined contribution from all three tethers $X_{T}, Y_{T}, Z_{T}, L_{T}, M_{T}$ and $N_{T}$. The axial tether force is regulated through the unstretched tether length $l_{u}^{i}$ defined for each tether in equation (15). Similarly, the moments on the shroud are generated by the combined tether forces in the fore and aft locations as a function of their position relative to the center of mass. These input forces can be related to the dynamics of each of the three independent winches located at the base station. The next paragraph discusses this point in detail.

\section{A. Actuator Dynamics}

The unstretched tether length $l_{u}^{i}$, seen in equation (14) is regulated by a DC motor winch speed input $u_{i}$. The DC motor is modeled as a first order transfer function defined with a time constant of $\tau$. A pure integrator is included to provide the desired change in unstretched length $l_{u}^{i}$ as a function of winch speed $u_{i}$ from its initial condition. There are three DC motors located on the base station and these are controlled independently of one another. The modeled winch speed dynamics for a single tether is shown in Figure 6:

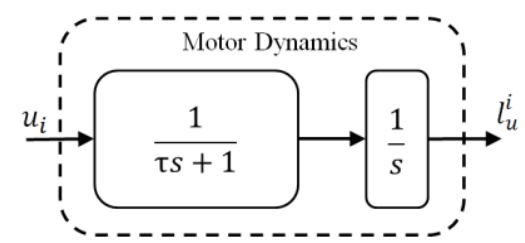

Figure 6 - Implemented Motor Winch Dynamics

\section{B. State Space Formulation}

The non-linear model is linearized using a first order Taylor expansion about a trim condition $x_{0}$. This consists of the body fixed velocities and positions related to the inertial frame of reference through appropriate Euler rotation matrices in roll, pitch and yaw. It is assumed that the tethers do not have direct control over the position in the $x$ and $y$ direction so the velocities $u$ and $v$ have been neglected in the linearization. The defined state vector is shown in equation (18) at a specific trim condition where $w_{0}, p_{0}, q_{0}, r_{0}$ are equal to zero and the altitude and pitch are linearized at set-points $z=z_{s p}$ and $\theta=\theta_{s p}$ respectively. The wind speed $v_{\text {wind }}$ at the point of linearization was assumed constant at $8 \mathrm{~m} / \mathrm{s}$ in this case.

$$
x=(w, p, q, r, z, \phi, \theta, \psi)
$$

At the defined operating point we have a state space model of the form:

$$
\dot{x}=A x(t)+B u(t)
$$

$$
y=C x(t)
$$

Where A, B and C are appropriate partial derivative matrices with the input vector being defined as

$$
u(t)=\left[u_{1}, u_{2}, u_{3}\right]
$$

Where, $u_{1}, u_{2}$ and $u_{3}$ are the three desired motor winch speeds of the fore, aft starboard and aft port DC motors respectively.

Manipulating equation (19) into transfer function form gives:

$$
X(s)=(s I-A)^{-1} B u(s)
$$

The linearized plant is initially unstable in open loop so must be stabilized before any additional control is applied. This was achieved using a state feedback law of the form $u=$ $-K x(t)$ where $\mathrm{K}$ is a $3 \times 8$ control gain matrix derived from applied LQR techniques. This results in a stable plant with stable poles that corresponds well with the full nonlinear model following a small perturbation about the initial state vector.

A simple multi-variable controller can then be designed to control the position and orientation of the shroud following a given step input. The two positions chosen for feedback control were the altitude and pitch, both of which are controlled through the winch speeds $u_{1}, u_{2}$ and $u_{3}$. The PID tuning method employed for multi-variable control is described in Sections C and D.

\section{PID Tuning Method}

Tuning of multivariable PID controllers for dynamic systems such as ships has been extensively studied in [15]. In this case, the Davison method was used to derive the integral gain of the system. The expression for the controller is given in (22):

$$
u(s)=K_{i} \frac{1}{s} e(s), \quad K_{i}=\varepsilon G^{-1}(0)
$$

$K_{i}$ is an integral feedback gain related to the inverse of the square open-loop transfer function matrix $G(s)$ evaluated at zero frequency. The scalar $\varepsilon$ is a tuning parameter. The system is tuned by starting at a small value of $\varepsilon$ then increasing it steadily to achieve the maximum speed of response for the closed-loop system.

The final controller is then derived using the PentinnenKoivo method. This alters the Davison method by including a proportional gain in the controller. The expression for the final controller is then given in (23):

$$
u(s)=\left(K_{P}+K_{i} \frac{1}{S}\right) e(s), K_{P}=p(C B)^{-1}, K_{i}=\varepsilon G^{-1}(0)
$$

The CB matrix employed is the closed form matrix after stabilization. The proportional gain matrix is then selected as a function of a proportional constant $p$ that is a constant scaling tuning parameter. Similarly, the integral gain is given by the response of the closed loop inverse plant dynamics at zero frequency and is modified by a constant tuning parameter $\varepsilon$. The derived gains have an inherent dependency on each control error which results in extreme coupling between both altitude and pitch set-points. 


\section{RESULTS}

To test the multivariable controller, the linearized system was subjected to two step inputs in altitude and pitch. The setpoint for the desired pitch angle was 10 degrees which is known as the optimum pitch angle for aerodynamic performance [13]. The simultaneous response of the system to two step inputs in altitude and pitch is shown separately in Figures 7 and 8 . In both cases it can be seen that the controller tracks the reference point well and at a good response rate. The disturbance rejection properties can also be seen at $\mathrm{t}=300 \mathrm{~s}$ when a step input was applied. Following the disturbance the system returns to a steady state in both altitude and pitch within 40 seconds.

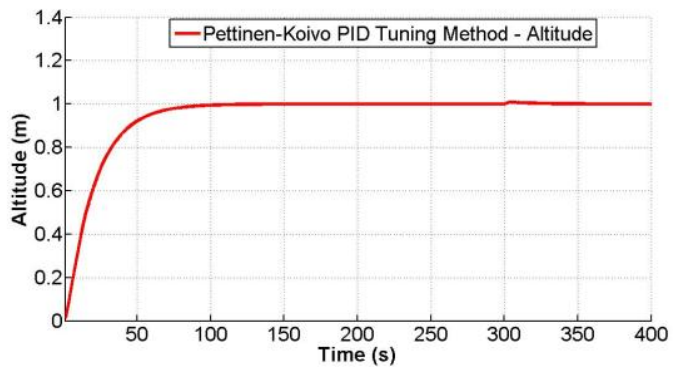

Figure 7 - Altitude vs Time

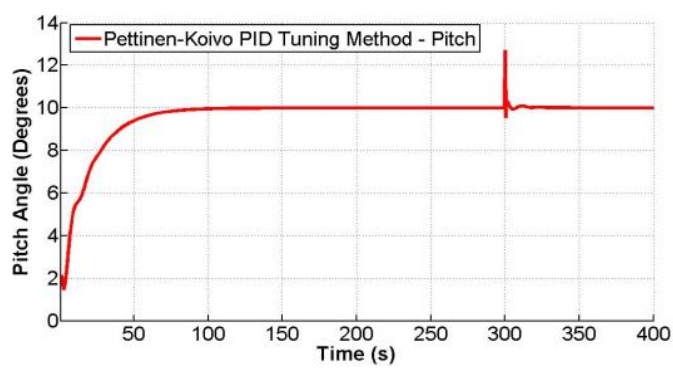

Figure 8 - Pitch Angle vs Time

\section{CONCLUSION}

This paper has sought to introduce a new form of wind energy systems known as Airborne Wind Energy Systems (AWES). A review of the different types of system and the control employed was shown in Section II. The complex trajectory control of kite and rigid body systems may make it complicated to enforce in real time applications. This is because the dynamic control relies on high aerodynamic efficiencies which may not exist in real time. However, there has been a lot of success in control development and testing in this area. In comparison to these systems a new lighter than air system developed by Altaeros Energies was introduced in Section III. Accounting for the significant aerodynamic nonlinearities present over the operating envelop will be important in the operation of this system. As such, a force ratio was developed to help define the transition points between buoyant to aerodynamic behavior on the shroud. Finally a linearized model was developed from the nonlinear Altaeros System and the Pentinnen-Koivo PID tuning method was employed. This resulted in a multivariable controller, controlling both pitch and altitude. It was seen that the controller works effectively and has a good response rate.
Future work will test these methods on the full nonlinear system and produce a working multivariable system that also includes regulation of the roll angle.

\section{ACKNOWLEDGMENTS}

The author wishes to acknowledge the support of Altaeros Energies without which this work would not be possible. This work has been funded by the EPSRC, project reference number EP/G037728/1.

\section{REFERENCES}

[1] C. L. Archer and K. Caldeira, "Global Assessment of High-Altitude Wind Power,” Energies, vol. 2, no. 2, pp. 307-319, May 2009.

L. Fagiano and M. Milanese, "Airborne Wind Energy: An overview," American Control Conference(ACC) 2012, no. 27-29 June, pp. 3132-3143, 2012.

"Makani Power." [Online]. Available:

http://www.makanipower.com/. [Accessed: 12-Apr-2012].

M. Loyd, "Crosswind kite power," Journal of Energy, vol. 4, no. 3, pp. 106-111, 1980

[5] M. Canale, L. Fagiano, and M. Milanese, "High Altitude Wind Energy Generation Using Controlled Power Kites," IEEE Transactions on Control Systems Technology, vol. 18, no. 2, pp. 279-293, Mar. 2010.

[6] L. Fagiano, M. Milanese, and D. Piga, "Optimization of airborne wind energy generators," International Journal of robust and nonlinear control, 2011.

[7] B. Houska and M. Diehl, "Robustness and stability optimization of power generating kite systems in a periodic pumping mode," Part of the IEEE Multi-Conference on Systems and Control, Yokohama, Japan, September 8-10, 2010, pp. 2172-2177, 2010.

[8] P. Williams, B. Lansdorp, and W. Ockesl, "Optimal Crosswind Towing and Power Generation with Tethered Kites," Journal of Guidance, Control, and Dynamics, vol. 31, no. 1, pp. 81-93, Jan. 2008

[9] L. Fagiano, "Control of Tethered Airfoils for High-Altitude Wind Energy Generation," Politecnico di Torino, 2009.

[10] M. Canale, L. Fagiano, and M. Milanese, "Power kites for wind energy generation," IEEE Control Systems Magazine, no. December, pp. 25-38, 2007.

[11] J. Samson, R. Katebi, and C. Vermillion, "A Critical Assessment of Airborne Wind Energy Systems," in IET Renewable Power Generation Conference, 2013, pp. 4-7.

[12] M. Nahon, G. Gilardi, and C. Lambert, "Dynamics/control of a radio telescope receiver supported by a tethered aerostat," Journal of Guidance, Control, and Dynamics, vol. 25, no. 6, 2002.

[13] C. Vermillion, T. Grunnagle, R. Lim, and I. Kolmanovsky, "ModelBased Plant Design and Hierarchical Control of a Prototype LighterThan-Air Wind Energy System, with Experimental Flight Test Results," American Control Conference, 2012

[14] J. Samson, R. Katebi, and R. C. Building, "A key design consideration for a Lighter than Air Wind Energy System," in The Science of making Torque from Wind Journal of Physics: Conference Series, 2014.

[15] P. Martin and R. Katebi, "Multivariable PID tuning of dynamic ship positioning control systems," Proceedings of IMarEST - Part A Journal of Marine Engineering and Technology, pp. 11-24, 2005.

[16] I. K. U.Kalabic, C.Vermillion, "Reference Governor Design for Computationally Efficient Attitude and Tether Tension Constraint Satisfaction on a Lighter-Than-Air Wind Energy System," European Control Conference, 2013. 